\title{
What does the Shinyei PPD42NS Low-Cost Dust Sensor Really Measure?
}

\author{
Michaël Canu, Boris Gálvis, and Malika Madelin
}

\begin{abstract}
Deteriorating air quality is of great concern around the world. Recently, citizen scientists, researchers, and many others have used low-cost devices such as the Shinyei PPD42NS dust sensor to measure particulate matter pollution in both developed and under-developed countries. However, few articles exist specifically on the features and performance of these sensors. Some have shown mixed results in terms of precision, accuracy, and repeatability, especially for portable applications. Frequently, users assemble the electronics and the sensors applying simple guidelines, using electric schematics, and coding extraneous algorithms to get questionable data. There is a need to better understand how it works exactly, its limitations and the effect of the program used to interpret the outputs of this sensor. This article provides a short electronic analysis of the Shinyei PPD42NS dust sensor and shows that the internal sensor electronic design (filters and detection stage) as well as the used data processing algorithm, limit its precision and accuracy by generating nonlinearities and biases. These issues avoid some applications like moving ones and imply that the algorithm used to process the sensor signals must be clearly presented in future articles.
\end{abstract}

Index Terms-Low-cost sensors, PM sensors, atmospheric aerosols, air pollution.

\section{INTRODUCTION}

Air quality measurement can greatly benefit from accurate but inexpensive measuring instruments to expand spatial coverage and bridge gaps between personal exposure and ambient concentrations [1], [2]. This is especially true for cities in low-income countries, given the higher levels of air pollutants they endure and the smaller budgets for air quality monitoring they spend. Budgets for research and environmental issues could be 100 times smaller in developing countries (for example, in France the environment ministry budget 2019 is 34.2 bn euros and the total government budget is 229 bn euros, which represents $15 \%$, in Colombia, for example, it is $0.14 \%$ ). Many civic initiatives on air quality monitoring like Air Casting [http://aircasting.org/] in USA, SIATA [https://siata.gov.co/] in Colombia or Air Citizen [http://aircitizen.org/] in France, have sprouted in recent years [3]. This could explain the increasing demand for low or ultra-low-cost dust sensors like

Manuscript received March 10, 2020; revised November 12, 2020. This work was supported in part by the university El Bosque.

Michaël Canu is with the University El Bosque, Electrical Engineering Department, Cra 9 N 131A-02, Colombia (e-mail: mcanu@unbosque.edu.co).

Boris Gálvis is with La Salle University, CLIMA group, Carrera 2 No 10-70, Colombia (e-mail: bgalvis@unisalle.edu.co).

Malika Madelin is with the PRODIG Lab, Universite de Paris, 5, cours des Humanités 93322 Aubervilliers Cedex, France (e-mail: malika.madelin@univ-paris-diderot.fr). the Shinyei PPD42NS. However, literature shows variable results in terms of precision and repeatability for this kind of sensor. Some authors [4]-[14] showed good correlations can be obtained between this kind of sensors and some of the most commonly referenced optical instruments like Dust-Trak, DustMate, Dylos, or TEOM (Tapered Element Oscillating Microbalance) or BAM (Beta Attenuation Mass) devices for prolonged exposure and stationary use. However, the Shinyei low-cost dust sensor output is either noisy, inaccurate, or non-linear for short time exposures and in both urban environments [6]-[8] and low particle concentrations [8], [9], [15], [16], humidity-dependent [6], and particle-size-dependent [11]. This, especially in the case of new portable applications, [17] leads to poor accuracy and noisy, unusable data. It is challenging to find a common pattern between data accuracy and a condition of use (humidity, temperature, particle source, etc.) that allows the user to predict the sensor measurement accuracy for a given experiment. In fact, few articles analyze how the Shinyei PPD42NS dust sensors functions in different conditions. While some studies conduct thorough evaluations [6], [8], [11], [18], some others describe approximate or inexact functioning information (http://aqicn.org/sensor/shinyei/). The problem is that many times, users, including re-searchers, simply reuse electric diagrams and algorithms directly downloaded from some websites without any adaptation (like in [19]). Many sensor users use data approximations based on linear regressions to get data from the Shinyei sensor, which works well for some applications, but, due to the lack of understanding of the sensor operation, it is impossible to extend those results to other situations. This article aims at clarifying the internal functioning of the Shinyei PPD42NS dust sensor and gives recommendations on how to improve, as far as possible, its performance for a variety of user applications.

\section{THE SHINYEI PPD42NS SENSOR}

\section{A. Dust Sensor Technologies}

Methods to measure particulate matter are based on four principles: gravimetric, mechanical, radioactive, and optical method. Each method can give different information and should be selected carefully, according to its intended application. The mechanical and filter-based gravimetric methods directly measure the mass of dust particles in a given volume of air. The gravimetric method is a U.S. Federal Reference Method [20]. The optical and radioactive methods measure the number of particles in a given volume from the Mie diffusion theory [21] and from the $\beta$ 
absorption/attenuation phenomenon, respectively. To obtain mass concentrations from these last two methods the number of particles must be converted into mass by assuming the particle density. There are also artifacts related to the chemical composition of the particles, which affect its refraction or diffusion properties, and its hygroscopicity. Moreover, the airflow stability inside the sensor can also affect the particle concentration estimation [22].

Sharp GP2Y1010AU0F, Shinyei PPD42N(S or J) and PPD60PV-T2, Syhitech DSM501A, Samyoung DSM501 or Amphenol/Telaire SM- PWM-01A are the most common used ultra-low-cost dust sensors in citizen proyects, and all of them use the same optical measurement principle. The Shinyei PPD42NS sensor seems very popular due to its very low cost (\$ 8 - 15 USD) and apparent ease of use. Note that the Shinyei PPD42NS, Syhitech DSM501A and Samyoung DSM501 sensors are identical and produce the same PWM-like output (Pulse Width Modulation signal with variable frequency and duty cycle). Therefore, this analysis also applies to these three sensors.

\section{B. Electronic Description}

Allen [1] wrote a technical document (reverse engineering report) on the Shinyei PPD42NS sensor, and this article aims to supplement this work. The Shinyei PPD42NS sensor is based on the optical diffusion detection of dust particles that pass through it, between a light source, an infrared light emitting diode (LED), and a photodiode detector (Fig. 1, from

http://www.takingspace.org/make-your-own-aircasting-parti cle-monitor/). An air flow between two openings in the plastic cover (called "Intake" and "Exhaust" in Fig. 1) is produced by a power resistor that heats the air in the optical chamber.

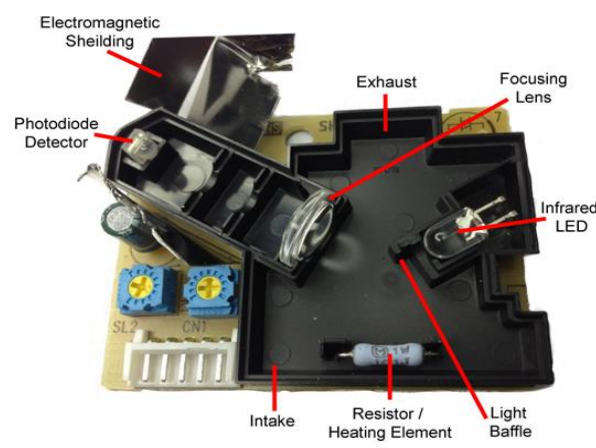

Fig. 1. Internal components (from Michael H., "Taking Space”, October, 2013,

http://www.takingspace.org/make-your-own-aircasting-particle-monitor/). Note: The top plastic cover was removed.

The detection stage is followed by two filters based on a general purpose, single-supply, bipolar operational amplifier (OAMP) model NJR/NJM2902. Dust particles modulate the current that passes through the photodiode and, consequently, the voltage at the point referenced TP16, as showed Fig. 3, for small size particulate matter (here, incense smoke). The steady state of this signal is about $1.52 \mathrm{~V}$ but depends on the supply voltage (here, $4.66 \mathrm{~V}$ ), and changes positively when particles pass through the sensor, due to the light diffusion.

After the detection stage, the photodiode signal passes through two filters that constitute a 2 nd-order band-pass filter, whose response is shown Fig. 4. The maximum gain of this filter is $616.6(55.8 \mathrm{~dB})$ and the minimum phase shift is at 5.7 Hz. The filter attenuates frequencies lower than $0.135 \mathrm{~Hz}$ and higher than $1175 \mathrm{~Hz}$, as calculated by Canu et al. [22]. The two following OAMP cells constitute two inverted comparators that produce the two output pulse signals. Each possess its own threshold voltage named Vref1 and Vref2 in Fig. 2. Without any modification, those references are set to 0.196 Vin for P1 and 1/2 Vin for P2. In addition, the user can adjust the P2 threshold voltage, either by connecting an external resistor or applying a given voltage between the pin "thresh" (sensor pin 5) and ground reference.

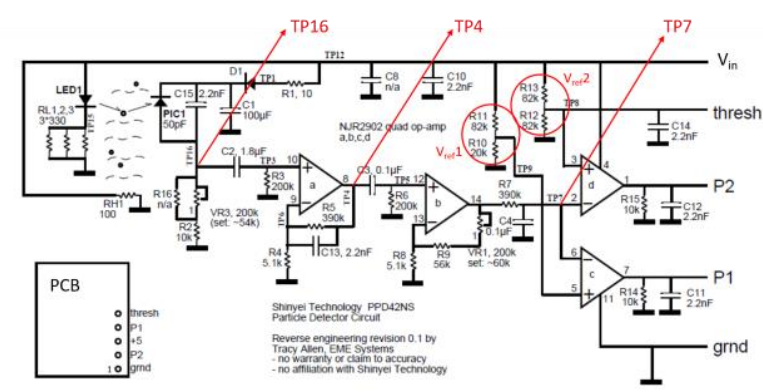

Fig. 2. Diagram of the PPD42NS sensor from Allen, T., with test point location. (C) 2018 IEEE. Reprinted, with permission, from Canu et al.

\section{Functioning Principle}

Canu et al. [22] have described the sensor functioning that is resume in Figure 3 and 4: the photodiode signal (red), the first filter's output (blue), the second filter's output (green) and the outputs P1 (purple) and P2 (cyan) for small concentration particles from incense smoke are plotted. All the experimental data shown in this article were collected through a National Instrument data acquisition card (DAC) USB-6211. The last stage of the circuit compares the filter output signal with the two threshold voltages. If the filter output voltage is higher than $0.196 \times$ Vin, then pin $\mathrm{P} 1$ passes from high level to low level and if the output voltage filter is higher than $0.5 \times \mathrm{Vin}$, then pin P2 passes from high level to low level. Figures 3 and 4 show real, low, and high signals, from incense smoke particles: TP16 input signal (blue curve) and TP7 output filter signal (red curve). P1 output level (purple curve) changes from high to low when TP7 signals overtake $0.98 \mathrm{~V}$ (the threshold voltage). When the TP7 signal goes below this same level, the output P1 goes back to the high ideal state (Fig. 3-Fig. 8).

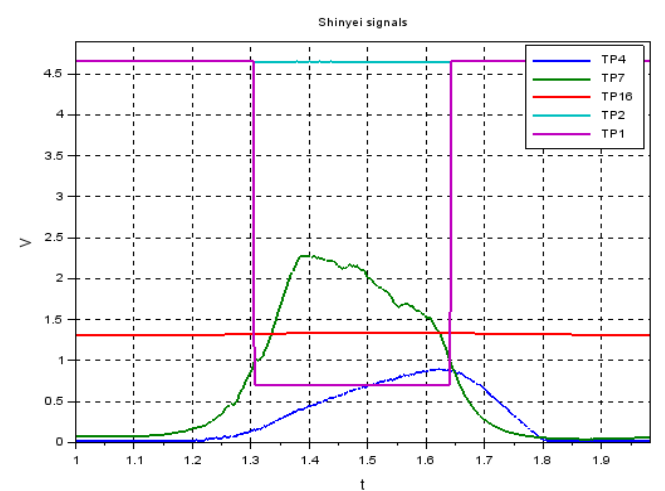

Fig. 3. Internal signals example (photodiode signal in red, first filter output in blue, second filter output in green, TP1 output in purple and TP2 output in cyan). 


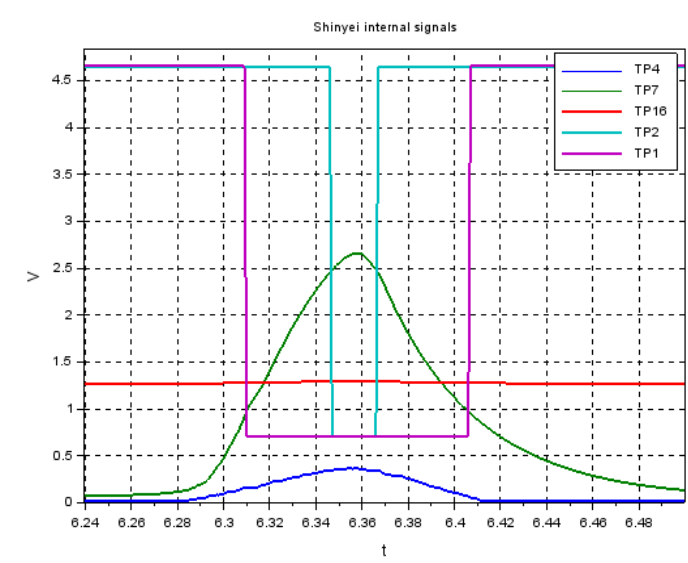

Fig. 4. Internal signals example (photodiode signal in red, first filter output in blue, second filter output in green, TP1 output in purple and TP2 output in cyan).

The manufacturer has settled the threshold voltages for the two outputs: P1 is supposed to give information about particles size over $1 \mu \mathrm{m}$ and $\mathrm{P} 2$ about particles over $2.5 \mu \mathrm{m}$ (information from Shinyei [23]).

\section{The Low Pulse Occupancy Time}

$\mathrm{P} 1$ or $\mathrm{P} 2$ pulse generation is related to the particle size because Mie diffusion intensity depends on particle size [3]. According to Shinyei [23], P1 or P2 low pulse duration is supposed to be related to the number and size of dust particles that pass through the sensor (see fig. 8 in [24]), thus, to the PM concentration. Moreover, the sum of P1 (or P2) low pulse duration in a given time, for example $30 \mathrm{~s}$, is considered to be quasi-proportional to the concentration of dust particles (Fig. 9, in [24]): the ratio between the low output duration and the reference time is called Low Pulse Occupancy time (LPO).

But what really represents the LPO measure?

\section{LPO MEASUREMENT ISSUES}

In fact, various phenomena can affect the LPO measure, as shown in the following. We can categorize those issues in two: issues that originate from the sensor principle ( $A$ and $B$ ), and electrical issues $(C, D$ and $E)$.

\section{A. LPO Non-linear Proportionality}

We call here "event" a "detection event": when the photodiode output presents a variation from the steady state, that is to say, when something - a particle or a group of particles - passes between the diode and the photodiode. The proportionality postulated between each event duration (large or small particle detection) and the LPO time is not valid for a wide range of event durations. Fig. 5 and 6 show two events: a $56 \mathrm{mV}$ pike (original photodiode signal) signal during 500 $\mathrm{ms}$, called Event 1 (Fig. 5), and a $57 \mathrm{mV}$ pike signal during $700 \mathrm{~ms}$, called Event 2 (Fig. 6). As can be seen, the LPO time is $290 \mathrm{~ms}$ for Event 1 and $185 \mathrm{~ms}$ for Event 2. Hence, for these two similar events, the sensor gives two different outputs that differ by $100 \mathrm{~ms}$. In fact, this unexpected result comes from the behavior of the two filters: the first signal Event 1 can be considered as a lower frequency signal than the second one (the first signal slope is lower). In summary, the high-pass filter induces a limitation for rapid input signal variations.

This phenomenon brings a problematic non-linearity to the LPO signal output because the LPO time is not always proportional to the detection signal duration (from the photodiode) so, to the size or number of particles.

\section{B. Particle Size Discrimination Error}

Because each output gives information about the particle size above 1 or $2.5 \mu \mathrm{m}$, according to Shinyei [23], the number of particles with diameter lower than $2.5 \mu \mathrm{m}$, called PM2.5, is given by the subtraction of P2 LPO from P1 LPO. Nevertheless, this is only a rough approximation because, on the one hand, the sensor is unable to differentiate a large particle from a set of two or more small particles and, on the other hand, this only gives the concentration for particles between 1 and $2.5 \mu \mathrm{m}$ (that is not equivalent to PM2.5). Hence, a non-trivial algorithm needs to be used to provide a good estimation of the particle concentration (see part IV).

Moreover, as denoted in [25], the relation between LPO duration and particle size is not very clear. In fact, Fig. 3 and 4 shows two event signals (in red, green, and blue) and the corresponding outputs (in cyan and purple). The first event (Fig. 3) produces a higher-level signal (TP4, in blue) than the second one but produces only a P1 output pulse (purple) while the second event (Fig. 4) produces a low pulse for both P1 and P2 (purple and cyan). A basic output treatment leads us to consider the second event as a large particle detection (> $2.5 \mu \mathrm{m})$ and the first event as a small particle detection (> 1 $\mu \mathrm{m})$. In fact, the first event reached a higher level than the second did, but during a longer time. Therefore, the derivative of the detection signal is lower in the first case than in the second one. This does not necessarily mean that the first event corresponds to a smaller particle than the second one. Furthermore, as reported by various authors [8], [11], [26]-[28], the incense or cigarette smoke used in various experiments is composed of particles inferior to $2.5 \mu \mathrm{m}$, with a peak from 20 to $650 \mathrm{~nm}$. Thus, the P2 output should not produce any low pulses, and $\mathrm{P} 1$ output should only produce few low pulses. This is not the case. So, it can be inferred than the P1 and P2 outputs also give information about particulate matter below $1 \mu \mathrm{m}$ and then should be taken into consideration in a specific manner (not only for size discrimination).

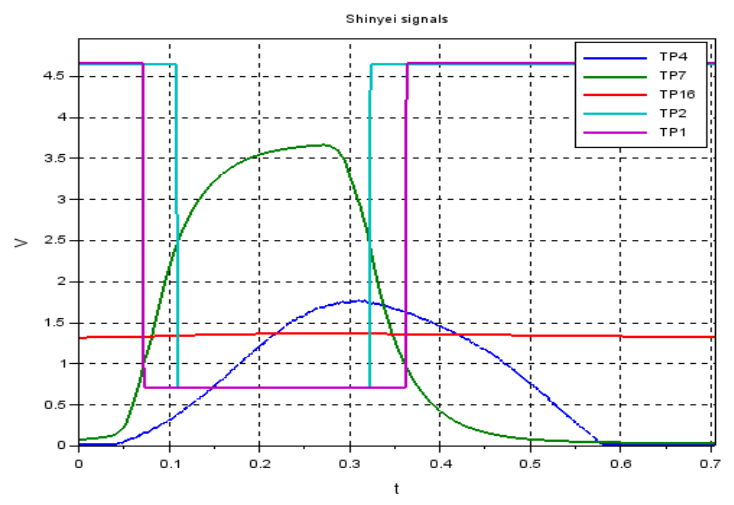

Fig. 5. Internal signals when a short event occurs.

\section{Supply Variation}

As mentioned by Canu et al. [22], the two threshold 
voltages are supply dependent, so the outputs pulse duration is also supply-dependent. In fact, because the threshold voltages come from voltage dividers (red circles in Fig. 2), any supply drop gives a threshold drop in proportion of the divider ratio. For example, if the supply voltage drops by $5 \%$, the P2 thresh-old drops by $2.5 \%$. Hence, the LPO time of either $\mathrm{P} 1$ or $\mathrm{P} 2$ is susceptible to change without any relation to the particles size or number, for which reason it is highly recommended to filter the power supply $V_{\text {in }}$ (in Fig. 2) by a large capacitor.

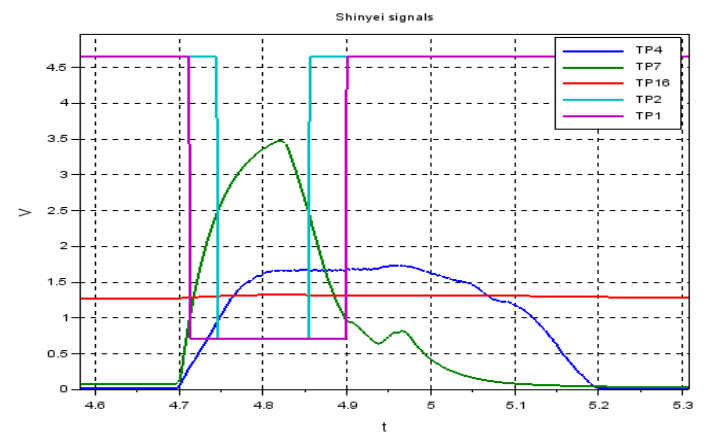

Fig. 6. Internal signals when a large event occurs.

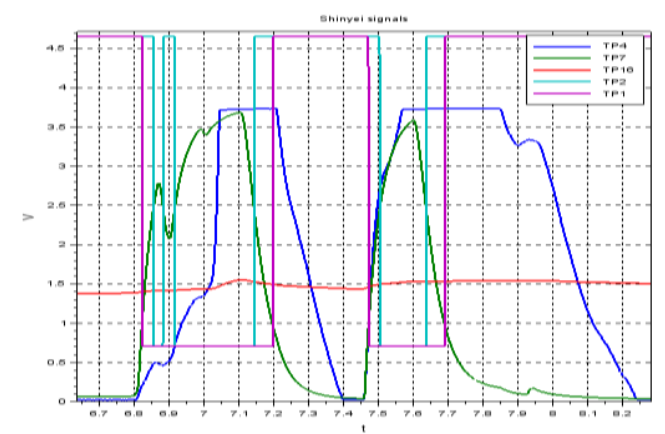

Fig. 7. Internal signals in case of OAMP saturation (in blue).

\section{OAMP Saturation}

Another non-linearity source appears in the internal sensor signals, which is an "unexpected" saturation effect from the OAMP. Fig. 7 shows an example of this phenomenon in case of high particles concentration (from incense smoke). In the two cases, the input signal from the photodiode reaches a high level and so the corresponding output of the first filter. Due to this high level and the filter gain, we can observe the saturation of the OAMP output (blue curve). In fact, this is not unexpected because the OAMP used by Shinyei present a Maximum Output Voltage Swing of 3.5V like the others low-cost OAMP in this category (LM324-like). Hence, it is an inherent characteristic of this sensor and nothing can be done to limit this phenomenon. Because the saturation occurs between the two filters, this induces a duration limit from the second filter. For this filter, the signal seems to be a step and the high-pass cell cuts this apparent continuous signal according to the step response of such a filter (green signal drops in Fig. 7). As in the previous case, this behavior brings a non-linear response between particles detection and LPO time.

\section{E. Output Oscillations}

Another common unexpected phenomenon that could occur is presented in Fig. 8. When the filter output signal reaches a level close to the threshold level, some oscillations appear (independently of the input signal from the photodiode). In the presented case, each output state change lasts about $5 \mathrm{~ms}$ : this, depending on the method used to measure the LPO time, could make the total event duration equal to zero if the program lasts more than $5 \mathrm{~ms}$ to compute a loop. Hence, in this case the total information is lost.

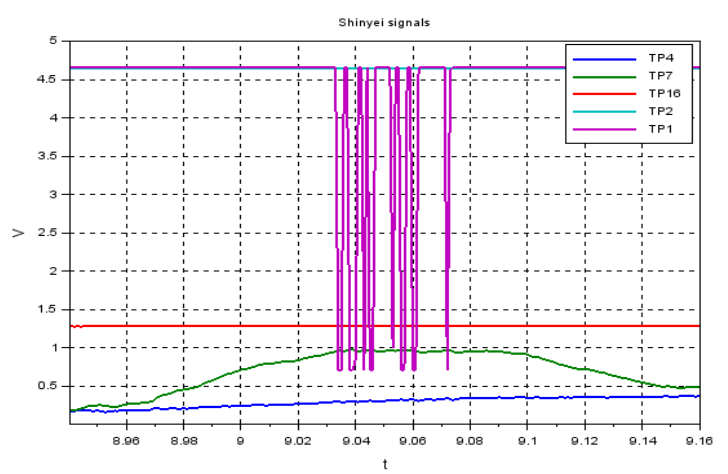

Fig. 8. Internal signals with P1 output oscillations.

\section{LPO MEASUREMENT ALGORITHM ISSUES}

In the literature, many articles since 2015 have shown results from the PPD42NS alone or comparison between the PPD42NS and other sensors [4]-[14], [16], [18], [19], [25], [29]. None of those articles present or give any precise information about the first stage of data processing. However, the sensor does not directly give the particulate matter concentration, unlike more sophisticated low-cost sensors (from manufacturers like Nova or Plantower). As illustrated in fig. 9, the PM concentration estimation (I4 column) is the result of a processing chain from the particulate matter detection (I1) that passes through 3 different treatment stages (F1, F2, F3). So, the PM estimation stage (F3) used to calculate the particulate matter concentration from the LPO ratio, and the program code that computes LPO ratio from sensor outputs (P1, P2 or P1 and P2, stage F2) impact the whole measure. Hence, if authors do not communicate about the program code and the output wiring, the findings could be hard to reproduce in the same or other contexts (independently of the PM estimation).

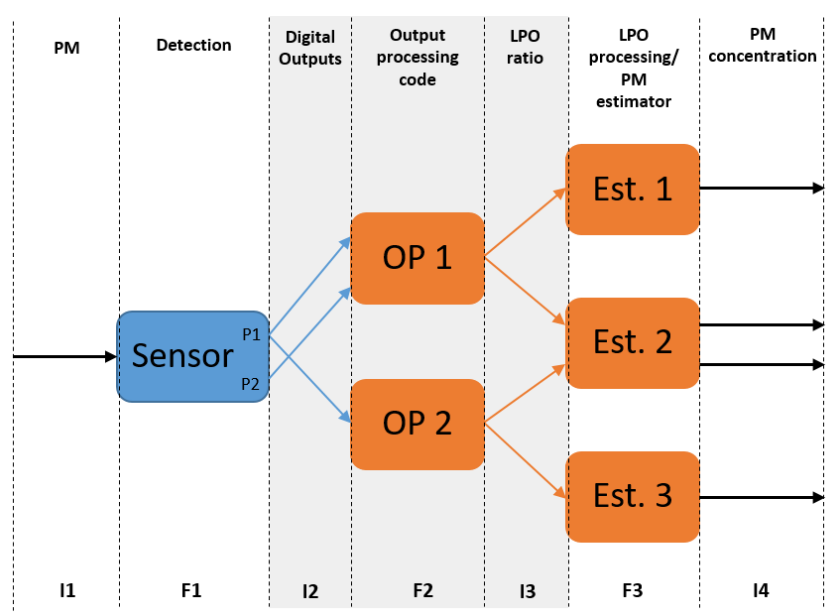

Fig. 9. Data processing flow from detection stage to PM concentration estimation (in grey, the focus of the present study). 


\section{A. LPO Measurement Algorithms}

We found two main methods in the literature to measure the LPO for this sensor (represented by OP1 and OP2, at stage F2 in Fig. 9). The first, measures the LPO directly using C code: We call this the Direct Measure Method (DMM). Projects like Luftdaten en Europe (https://luftdaten.info/) use this method. The second, uses a built-in function like pulseIn(), called here Indirect Measure Method (IMM) which is only available for Arduino@-based platforms, (see AirBeam project https://github.com/HabitatMap/AirCastingAndroidClient/bl ob/master/arduino/aircasting/aircasting_shinyeiPPD42NS.in o). It is important to note that only the DMM allows an adequate treatment for both $\mathrm{P} 1$ and $\mathrm{P} 2$ outputs. The use of the built-in function pulseIn() works only for one of the two outputs. As indicated in Shinyei's application note [23], to calculate the PM2.5 concentration, the two outputs should be used. Therefore, if only P1 output is used [6], all the particles with size over $1 \mu \mathrm{m}$ will generate a low pulse, hence, this cannot give a pretty correct PM2.5 concentration estimation. However, it is a common method especially in case of Arduino $@$ processor implementation, which is a common low-cost platform for citizens projects but also for many students' projects.

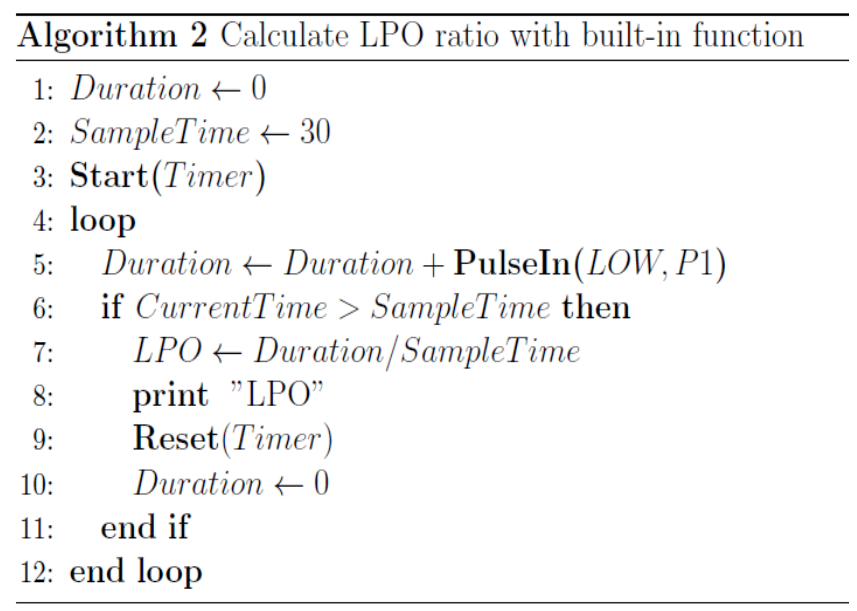

Fig. 10. The IMM code.

\section{B. Direct Measure Method}

The DMM computes the duration of the two outputs during a given sample time, detecting the low-to-high and high-to-low changes of both signals. Fig. 10 shows the DMM algorithm: if the code detects the beginning of an "event", that is to say an high-to-low change on P1 or P2 input, a particle (or a group of particles) has passed through the detection device, so the current time is recorded (lines 11 and 16). If a low-to-high change is detected, then the event is ending, and the code measures the time elapsed since the beginning of the event (lines 13 and 18) by adding to the duration the rest of the current time to the starting time. When the sample time is reached (line 20), the LPO ratio is calculated by dividing the cumulative duration of the events by the sample time. This ratio, between 0 and $100 \%$, can then be used to calculate the particles concentration from a calibrating curve provided by the manufacturer (like in [23]) or found experimentally (like in [4], [5], [9], [14]).

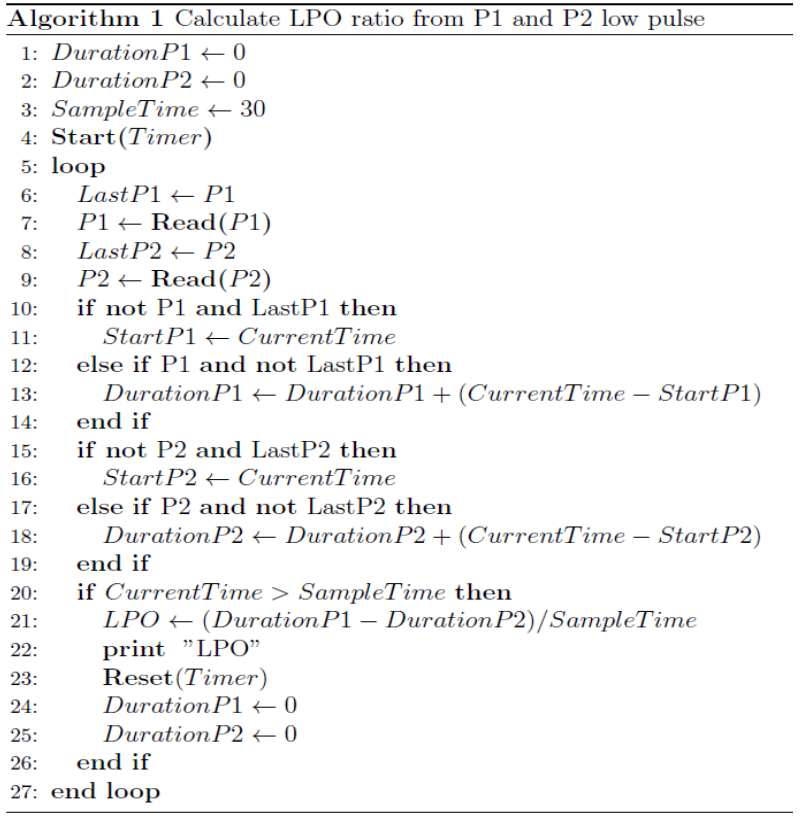

Fig. 11. The DMM code.

\section{Indirect Measure Method}

The IMM uses a built-in Arduino $@$ (this is a very popular development platform based on ARV processors) function, generally pulseIn(), to detect and measure the P1 or P2 low pulse. After the measure, as shown in Fig. 11, the current low pulse duration is a sum of the total duration and when the sample time is reached (line 6); then the algorithm computes the LPO as in the precedent case.

The main problem here is that this built-in function cannot process more than one input at the same time: $\mathrm{P} 1$ or $\mathrm{P} 2$. The second problem is that during the function processing, it is not possible to execute any other instruction because the function code waits for the end of the pulse before returning the duration to the main code. Moreover, if the built-in function is called during a pulse, then the internal code waits for a low-to-high signal before starting the detection routine and, therefore, only the next pulse will be processed. In this case, the current pulse is lost.

\section{Measure Method Impact Evaluation}

The two methods give inaccurate LPO measurements when the algorithm measurement period expires during an output low pulse. In this case, the current low pulse duration is lost. Generally, this issue is untreated but can be easily fixed by detecting it, summing up the current elapse time in the current period and then restarting the timer for the next event. Off course, this has lower significance for large measurement intervals like $30 \mathrm{~s}$ or more. However, because many new mobile applications require short data acquisition time (1-3 s for example), the significance of this issue increases because the ratio between LPO time and sample time also increases. The impact of this could be different if the user implements IMM or DMM code, generating a random pulse counting, so an uncertain LPO measure, especially for low sample times.

To evaluate this differential impact between IMM and DMM methods according to the sample time, we conduct a comparative experiment. In fact, we want to compare the LPO ratio measure from P1 for the IMM method, and from 
P1-P2 for the DMM method (the grey zone from I2 to I3 in Fig. 9). So, we must compare raw data (LPO ratio) and not processed data (like PM concentration estimation) to not adding the PM estimation effect. So, a calibration or a monitoring with a precise reference sensor/apparatus is not necessary during the test since it is a relative comparison.

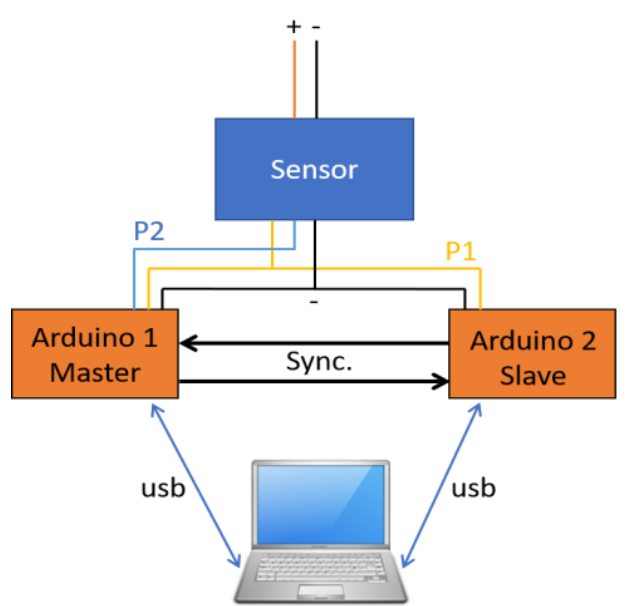

Fig. 12. Dual measure experiment.

The difference in LPO measurement through these two methods was evaluated connecting two identical processors (two Arduinos $($ Pro microprocessors, Fig. 12) to the same sensor and used synchronized programs to measure sensor output at the same time (Grafcet algorithm Fig. 14), one runs as master and the other one as slave.

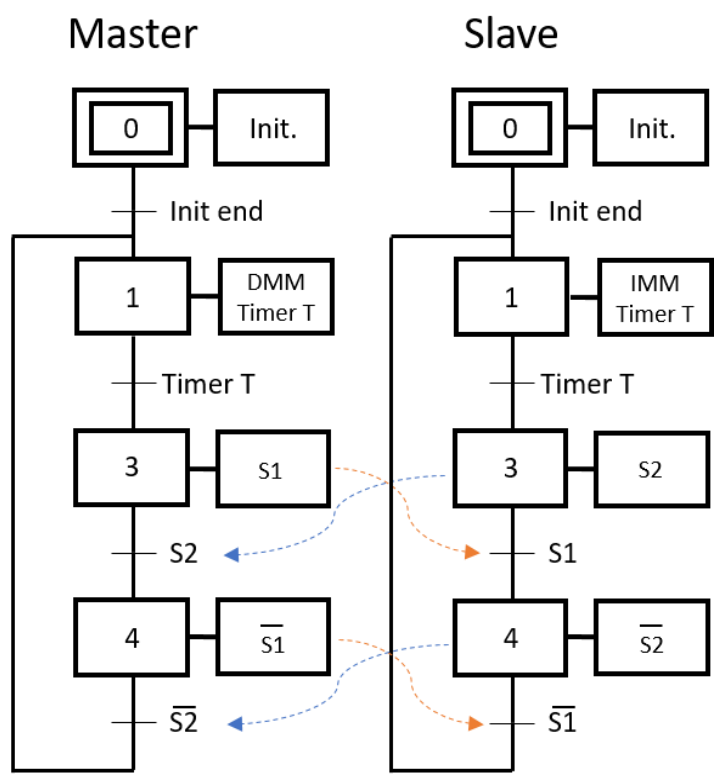

Fig. 13. Algorithm used to synchronize the two Arduinos.

The programs used are the same as in AirBeam project [30] for IMM and as in Luftdaten Project [31] for DDM, with a software/hardware barrier synchronization algorithm [32] to ensure an identical start time for the LPO measurement (fig. 13). In fact, each processor uses the same sample time between 1 and 30 seconds, but with an independent clock. So, the barrier algorithm is necessary to get the same event as measure starting point. The sensor was placed in a vertical position in a $0.08 \mathrm{~m} 3$ plastic box and after the recommended warm-up time [22], incense smoke was introduced or filtered with an EPA filter to control the PM2.5 concentration in the box. The particle concentration was monitored with a Plantower PMS5003 low-cost sensor to guarantee at least three variations between 5 and $600 \mu \mathrm{g} / \mathrm{m}^{3}$ during each test. The PMS5003 sensor gives an underestimated PM2.5 measure by a ratio of 1.6 (the factor was found comparing the Plantower measure to a Gillian air sampling pump measure, from five $24 \mathrm{~h}$ tests in the street) but with a good accuracy reported by previous studies with this kind of sensors [15], [33]. However, the PMS sensor was only used to control the variations limits of the concentration into the box and not the exact concentration, so a more precise calibration was not required. As mentioned in [26]-[28], the distribution of incense smoke, like cigarette smoke [8], is centered on nanoparticles below $1 \mu \mathrm{m}$, so a low impact from large particles $(\geq 2.5 \mu \mathrm{m})$ was expected.

Table I shows that the correlation coefficient between DMM and IMM for the six experiments between $1 \mathrm{~s}$ to $30 \mathrm{~s}$ sample time ( $2^{\text {nd }}$ column) is always inferior to 1 . So, the IMM algorithm always computes an LPO ratio superior to the DMM algorithm ratio (fig. 15). This is quite surprising because the incense smoke does not contain large particles $(>2.5 \mu \mathrm{m})$. In fact, the ratio in the DMM case is: (P1 LPO time - P2 LPO time)/Sample time. So, this difference should be inferior to P1 LPO time only in presence of large particles, which is not the case with incense smoke.

Moreover, the difference between the Direct and the Indirect Method in P1 LPO ratio calculus lies essentially in the use of the pulsin(). Fig. 14 shows this difference, depending on the sample time. The P1 LPO time measuring by DMM algorithm is quite similar to the DMM one (with many null values) for small sample times. But for sample times over $5 \mathrm{~s}$, the DMM measure of P1 LPO time is on average superior to the IMM measure of P1 LPO time. This is essentially because the pulsin() function loses events, as said in part. VI.C. This is a direct LPO time measure method effect not yet reported in the literature.
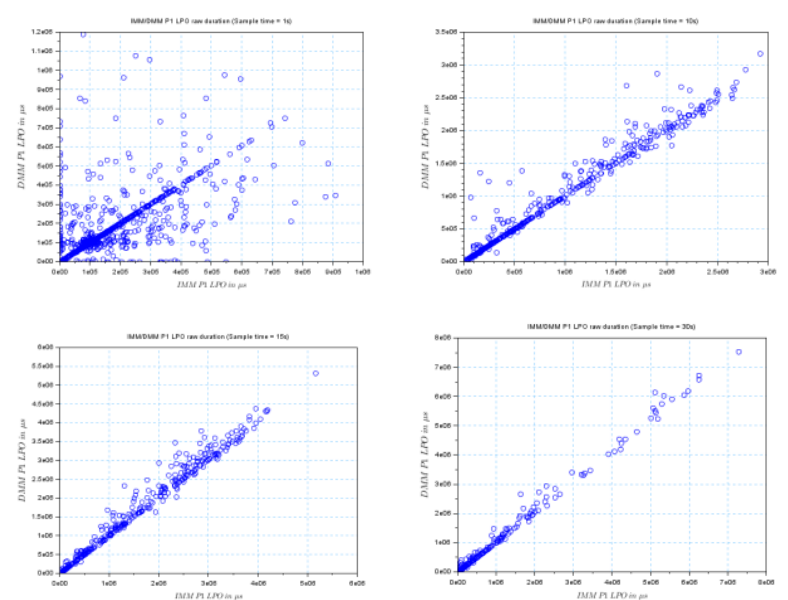

Fig. 14. P1 LPO time comparison (in $\mu \mathrm{s}$ ) with Direct and Indirect Measure Method for 1s (top left), 10s (top right), 15s (bottom left) and 30s (bottom right). We can see that data points are in average above the $y=x$ curve that indicates a P1 LPO time measure with DMM superior to the P1 LPO time measure with IMM.

We can also observe that the average ratio between IMM and DMM increases with the sample time (nearer 1 when the sample time increases), and the measure dispersion is always higher for the IMM and increases as the sample time 
decreases (Fig. 14). This is a direct combined effect of the detection/filtering process with the measurement algorithm. Below $15 \mathrm{~s}$, and especially around $1 \mathrm{~s}$ sample time, the two methods present different behaviors, which could drive to some impacts on the concentration evaluation results. However, below $30 \mathrm{~s}$, the IMM gives a measure that is more scattered than the DMM. This is coherent with the poor accuracy reported by the only author who has investigated the sample time impact on PPD42NS LPO measure [7, Fig. 3 p. 525]. So, depending on the algorithm used, it is necessary to adjust the method/function that evaluates the PM concentration to the sample time. This has never been properly discussed in the literature.

TABLE I: INFLUENCE OF THE SAMPLE TIME ON THE LPO MEASURE (N IS THE

\begin{tabular}{cccc}
\multicolumn{4}{c}{ NuMBER OF DATA POINTS) } \\
\hline Sample Time (s) & Corr. & Regr. DMM=f(IMM) $y=a x+b$ & $\mathrm{n}$ \\
\hline 30 & 0.993 & $0.97 x+0.09$ & 268 \\
15 & 0.978 & $0.89 x+0.4$ & 412 \\
10 & 0.981 & $0.95 x+0.57$ & 548 \\
5 & 0.938 & $0.76 x+0.93$ & 896 \\
3 & 0.827 & $0.70 x+1.28$ & 374 \\
1 & 0.58 & $0.44 x+5.63$ & 1500 \\
\hline
\end{tabular}
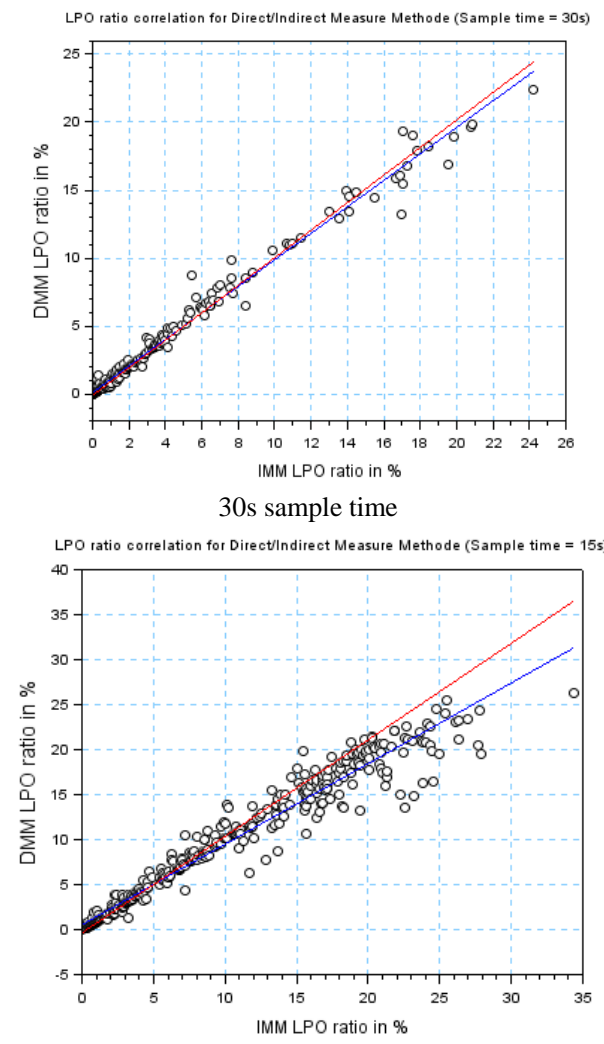

15 s sample time

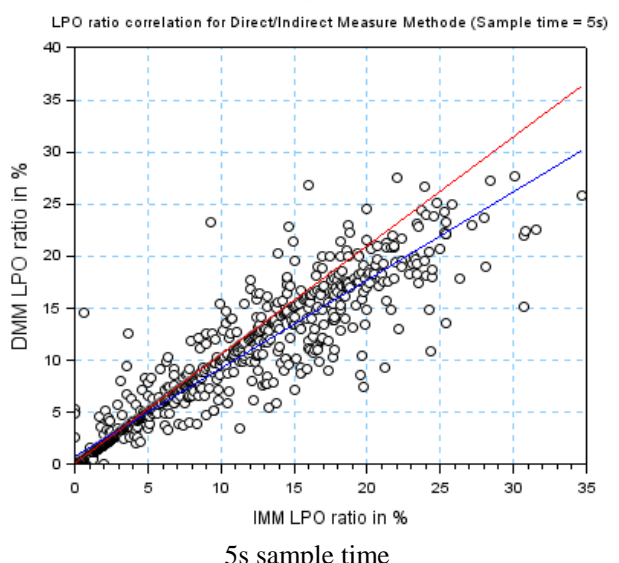

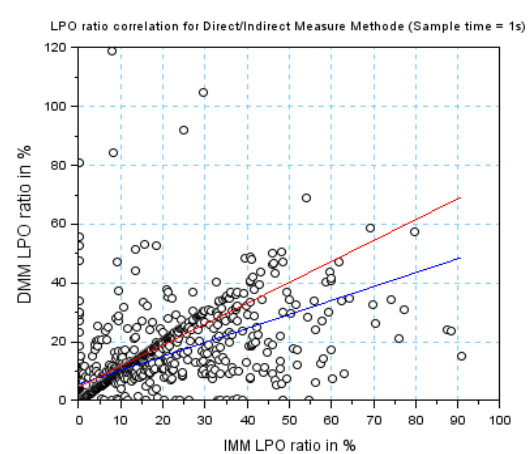

Fig. 15. IMM vs DMM method for 30, 15, 5 and $1 \mathrm{~s}$ sample time. In blue, the $\mathrm{DMM}=\mathrm{f}(\mathrm{IMM})$ linear regression and in red, the $\mathrm{IMM}=\mathrm{f}(\mathrm{DMM})$ linear regression. As we can see, the less the sample time is, the lower the correlation between the two methods is and the higher the data dispersion is.

\section{DISCUSSION}

As mentioned by some authors like Holstius et al. [7]; Johnson et al. [9] or Kelly et al. [33], the low-cost dust sensor Shinyei PDD42N could be appropriate for some stationary applications. For example, it can be used to measure indoor PM2.5 concentrations during large periods (with a $30 \mathrm{~s}$ or higher sample time). However, as demonstrated in this article and in Canu et al. [22], due to some inherent characteristics of it, many applications may require a careful implementation, complex calibration [6], [10] and other applications should be avoided. For example, the non-linear behavior of the sensor due to the filter characteristics and the algorithm used to compute the measure prevent accurate mobile applications. We saw that the algorithm used, which depends on the hardware platform, impacts the LPO time/ratio measure (in terms of magnitude and measure dispersion). Because it is impossible to process the two outputs of the sensor at the same time, it is not recommended to use faster built-in functions like pulsin() to measure the LPO time (IMM). Moreover, although the DMM is the reference method to process the two outputs of the sensor, it needs enough sample time to compute the LPO without losing information. It can be noted that those issues could also depend on the speed of the processor and the kind of programming language used (assembly code allows faster execution time than $\mathrm{C}$ code for example). However, in common situations (Arduino@-like platforms and $\mathrm{C}$ code) the sample time must be higher than 15 $\mathrm{s}$, and for better results, higher than $30 \mathrm{~s}$, to minimize the method effect on measure dispersion. For those reasons, it seems impossible to use it for mobile applications on bicycles, motorcycles, or cars. For example, the average speed for a bicycle ride is $16 \mathrm{~km} \mathrm{~h}-1$, so in $30 \mathrm{~s}$ the sensor travels $133 \mathrm{~m}$, which makes unprecise any study in an urban context. Additionally, as shown in Canu et al. [22], the air flow perturbation produced by the sensor movement (or some wind) could also affect the airflow through the sensor and so the output of the sensor. Finally, for static applications, as demonstrated by some authors [6], [10], [32], [33], it is possible to reach an acceptable accuracy by integrating the measure during a large operation time and using a suitable humidity correction. In some cases, the results are closed to those given by more sophisticated and expensive sensors, but present always a quite important error [7], [16] and require complex calibration (Artificial Neural Network [6], Gradient Boosting Model [10]). Adding a fan to the sensor could also 
improve its performance, guaranteeing a more precise response for low concentrations and eliminating null values at low concentrations.

\section{CONCLUSION}

Low-cost dust sensors, such as the Shinyei PPD42NS sensor, allow a wide-scale development of air quality measurement and monitoring projects. However, for accurate data collection, the users must pay attention to the mechanical and electronic implementation, as well as to the software processing used. Nevertheless, due to electronic characteristics, some information from the detection stage is lost during the signal conditioning. Any algorithm cannot compensate this loss of information nor any mathematical function outside the sensor. As demonstrated here, the data processing algorithm also impacts the LPO ratio measure; hence, this avoids a generalization of the results from an investigation to another one in an easy or "plug and play" manner if authors do not disclose any information about it. Consequently, in future research articles based on this sensor, details about algorithm used to process the data from the sensor should also be mentioned to facilitate the replication of the experiments with the same or different hardware platform. In particular, the number of outputs used (ideally P1 and P2), the method used to compute the LPO time, and the sample time should be presented.

\section{CONFLICT OF INTEREST}

"The authors declare no conflict of interest".

\section{AUTHOR CONTRIBUTIONS}

Michaël Canu wrote the paper, Boris Galvis gave access to its laboratory and experimental facilities; Malika Madelin participated in analyzing the data.

\section{REFERENCES}

[1] Y. Contreras et al., "Exposure to fine particulate, black carbon, and particle number concentration in transportation microenvironments," Atmospheric Environment, vol. 157, pp. 135-145, 2017.

[2] E. G. Snyder et al., "The changing paradigm of air pollution monitoring," Environmental Science and Technology, vol. 47, no. 20 , pp. 11369-11377, 2013.

[3] R. Williams et al., "Applications of low-cost sensing technologies for air quality monitoring and exposure assessment: How far have they gone?" Environnent International, vol. 116, pp. 286-299, 2018.

[4] T. T. Allen. (2013). De-construction of the Shinyei PPD42NS dust sensor. [Online]. Available: http://takingspace.org/wp-content/uploads/ShinyeiPPD42NSIDeconstr uction\TracyAllen.pdf

[5] E. Austin et al., "Laboratory evaluation of the Shinyei PPD42NS low-cost particulate matter sensor," PLoS ONE, vol. 10, no. 9, pp. 1-17, 2015 .

[6] L. Bai et al., "Long-term field evaluation of low-cost particulate matter sensors in Nanjing," Aerosol and Air Quality Research, vol. 20, no. 2 , pp. 242-253, 2020.

[7] D. M. Holstius et al., "Field calibrations of a low-cost aerosol sensor at a regulatory monitoring site in California," Atmospheric Measurement Techniques, no. 7, pp. 1121-1131, 2014.

[8] R. Jayaratne et al., "Low-cost PM2.5 sensors: An assessment of their suitability for various applications," Aerosol Air Qual. Res., vol. 20, pp. 520-532, doi: 10.4209/aaqr.2018.10.0390, 2020.

[9] K. K. Johnson, M. H. Bergin, A. G. Russell, and G. S. W. Hagler, "Field test of several low-cost particulate matter sensors in high and low concentration urban environments," Aerosol and Air Quality Research, vol. 18, pp. 565-578, doi:10.4209/aaqr.2017.10.0418, 2018.
[10] N. E. Johnson, B. Bonczak, and C. E. Kontokosta, "Using a gradient boosting model to improve the performance of low-cost aerosol monitors in a dense, heterogeneous urban environment," Atmospheric Environment, vol. 184, August 2017, pp. 9-16.

[11] D. Liu, Q. Zhang, J. Jiang, and D. R. Chen, "Performance calibration of low-cost and portable particular matter (PM) sensors," Journal of Aerosol Science, no. 112, 2017.

[12] M. Madelin and S. Duché, "Low cost air pollution sensors: New perspectives for the measurement of individual exposure?" presented at International Conference on Urban Climate, ICUC9, Toulouse, 2015.

[13] S. Sousan, K. Koehler, L. Hallett, and T. M. Peters, "Evaluation of consumer monitors to measure particulate matter," Journal of Aerosol Science, no. 107, pp. 123-133, 2017.

[14] Y. Wang et al., "Laboratory evaluation and calibration of three low-cost particle sensors for particulate matter measurement," Aerosol Science and Technology, vol. 49, pp. 1063-1077, 2015.

[15] T. Sayahi, A. Buttereld, and K. Kelly, "Long-term eld evaluation of the Plantower PMS low-cost particulate matter sensors," Environnemental Pollution, no, 245, 2019.

[16] L. Sheppard et al., "Calibration of low-cost particulate matter sensors: Model development for a multi-city epidemiological study," Environment International, no. 134, p. 105329, 2019.

[17] Y. Zhuang et al., "AirSense: A portable context-sensing de-vice for personal air quality monitoring," in Proc. the 2015 Workshop on Perva-sive Wireless Healthcare - MobileHealth'15, pp. 17-22, 2015.

[18] P. Sotirios, PM2.5 Device Comparison, $2016 .$.

[19] H. Pritchard, J. Gabrys, and L. Houston, "Re-calibrating DIY: Testing digital participation across dust sensors, fry pans and environmental pollution," New Media \& Society, vol. 20, no. 12, pp. 4533-4552, 2018.

[20] R. W. Wiener et al., "Federal reference and equivalent methods for measuring fine particulate matter," Aerosol Science and Technology, vol. 34, pp. 457-464, 2001.

[21] G. Mie, "Beiträge zur Optik trüber Medien, speziell kolloidaler Metall ösungen," An-nalen der Physik, vol. 25, pp. 377-445, 1908.

[22] M. Canu et al., "Understanding the Shinyei PPD42NS low-cost dust sensor," presented at 2018 IEEE International Conference on Environmental Engineering (EE), IEEE, Milano, pp. 1-10, 2018.

[23] T. Shinyei, "Particle size discrimination by PPD42NSJ," Technical Report, Shinyei Technology Co., Ltd., 2013.

[24] D. Brooks, Measuring Particulates in the Air Using Inexpensive Sensors, 2016.

[25] A. C. Rai and P. Kumar, "Summary of air quality sensors and recommendations for application," Technical Report 689954, University of Surrey, 2017.

[26] J. Jetter et al., "Characterization of emissions from burning incense," Science Total Environment, no. 295, pp. 51-67, 2002.

[27] X. Ji et al., "Caractérisation des particules générées par la combustion d'encens," 24ème Congrès Francais sur les Aérosols, Paris, p. 6, 2009.

[28] S. W. See and R. Balasubramanian, "Physical characteristics of nanoparticles emitted from incense smoke," Science and Technology of Advanced Materials, no. 8, pp. 25-32, 2007.

[29] I. W. Lenggoro et al., "Shinyei based sensor with added roof enhanced detection of indoor particulate matter," International Journal of Integrated Engineering, vol. 11, no. 7, 2019, pp 67-76.

[30] H. M. Besser, Air Beam Project, 2014.

[31] J. A. Lutz and D. Lackovic, Luftdaten Project, 2018.

[32] D. Hensgen, R. Finkel, and U. Manber, "Two algorithms for barrier synchronization," International Journal of Parallel Programming, no. 17, pp.1-17, 1988.

[33] K. E. Kelly et al., "Ambient and laboratory evaluation of a low-cost particulate matter sensor," Environnemental Pollution, no. 221, 2017.

Copyright (C) 2021 by the authors. This is an open access article distributed under the Creative Commons Attribution License which permits unrestricted use, distribution, and reproduction in any medium, provided the original work is properly cited (CC BY 4.0).

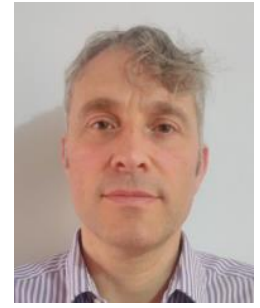

Michaël Canu is originally from France. M. Canu received a master's degree in automatic control from Ecole des Mines de Nantes, France, in 2007 and earned a Ph.D.'s degree in electrical engineering from Los Andes University, Colombia in 2015.

After a first engineering position in the Ecole des Mines in France, he earns a post-doctorate position at Los Andes University, and then he became an associate professor at the Electrical Engineering

Department of El Bosque University in Bogota, Colombia. 
He published an article in the IEEE International Conference in Environmental Engineering and a poster in the International Air Conference in 2018 on this same topic. He currently works on various approaches to model particulate matter distribution from partial data measures.

Pr. Canu is used to revise article for engineering education journals or conferences like Active Learning in Engineering Education (ALE) or in automatic control for Cogent Engineering.

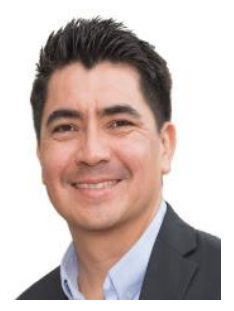

Boris Gálvis was born in San Gil, Colombia in 1975. $\mathrm{He}$ received his bachelor's degree in chemical engineering from Universidad Industrial de Santander (Bucaramanga, 1998), obtained a MSc in civil engineering from Universidad de Los Andes (Bogota, 2006); a MSc (Atlanta, 2010) and PhD (Atlanta, 2013) in environmental engineering from the Georgia Institute of Technology. Boris does research on the processes that emit, transform and deposit particulate matter and other air contaminants.

$\mathrm{He}$ is an associate professor at the Environmental Engineering Program of Universidad de La Salle in Bogota, Colombia. He has previously worked as a Consultant for several environmental government agencies in Colombia, doing research in air pollution.

His most recent work is "Personal exposure to air pollutants in a Bus Rapid Transit System: Impact of fleet age and emission standard". R. Morales Betancourt, B. Galvis, JM. Rincón-Riveros, MA. Rincón-Caro, A. Rodriguez-Valencia, O. L Sarmiento. Atmospheric Environment, Volume 202, p. 117-127, 10, 2019. "Development and Evaluation of a Comprehensive Atmospheric Emission Inventory for Air Quality Modeling in the Megacity of Bogotá". J. Pachón, B. Galvis, O. Lombana, LG Carmona, S. Fajardo, A. Rincón, Atmosphere 9 (49) 11, 2018 and "Exposure to fine particulate, black carbon, and particle number concentration in transportation microenvironments". R. Morales-Betancourt, B. Galvis, S. Balachandran, J.P. Ramos-Bonilla, O.L. Sarmiento, S.M. Gallo-Murcia, Y. Contreras. He has conducted many studies aimed to assess the impact from diverse mobile and industrial sources and resuspended dust, to understand particulate matter composition and to study air quality trends. A current focus of his research is to evaluate the exposure to air pollution in transport microenvironments and measure emission factors.

Professor Galvis has received the tower award from the Georgia Institute of Technology.

Malika Madelin was born in 1975 in France, who studied geography until a $\mathrm{PhD}$ in geography-climatology (2004), on the frost hazard in the Champagne vineyards (under the supervision of Prof. Gérard Beltrando, Université de Paris, Paris, France).

She became an assistant professor in geography at Université de Bourgogne (Dijon), then at Université de Paris. She teaches climatology and methods in geography (GIS, modelisation, spatial analysis and so on). Her topics are local climatology, urban climatology (Urban Heat Island, air pollution), citizen science (with low-cost sensors) and massive spatial data.

Dr. Madelin participates in review committees of Climatologie (http://lodel.irevues.inist.fr/climatologie/) and Hypergeo.eu (free-access electronic encyclopaedia devoted to the epistemology of geography). She is an ANSES expert (French Agency for Food, Environmental and Occupational Health \& Safety). 